\title{
Abnormal Mitochondria Organization and Oxidative Activity in the Palate Muscles of Long-Term Snorers with Obstructive Sleep Apnea
}

\author{
Per S. Stål ${ }^{a}$ Bengt Johansson ${ }^{b}$

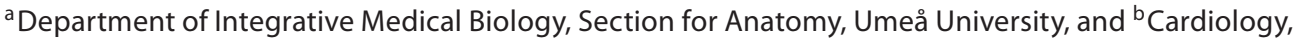 \\ Heart Centre, Umeå University Hospital, Umeå, Sweden
}

\section{Key Words}

Palate muscle • Mitochondria • Capillaries · Snoring •

Sleep apnea $\cdot$ Obstructive sleep apnea syndrome

\begin{abstract}
Background: Histopathological alterations and a reduced number of capillaries have been observed in the palate muscles of snorers with obstructive sleep apnea syndrome (OSAS). These changes may create a substrate for decreased microcirculation, impaired aerobic metabolism and muscle dysfunction and contribute to upper airway obstruction during sleep. Objectives: The aim was to analyze mitochondria distribution and oxidative enzyme activity in relation to capillary supply in the palate muscles of patients with a history of long-term snoring and OSAS. Methods: Palatopharyngeus (PP) and uvula (UV) muscle samples were obtained from 8 patients undergoing uvulopalatopharyngoplasty due to habitual snoring and OSAS. The muscles were analyzed with enzyme- and immunohistochemistry and morphometry. Results: Abnormalities in the internal organization of mitochondria and oxidative activity were observed in $39 \pm 15 \%$ of the fibers in the PP and $4 \pm 3 \%$ in the UV, but not in control samples. The majority of these fibers had a lobulated contour and trabecular internal organization of mitochondria. The number of capillaries around abnormal fibers (PP
\end{abstract}

$0.9 \pm 0.3$, UV $0.4 \pm 0.1$ ) was lower than in fibers of a normal appearance in both patients (PP 1.4 \pm 0.6 , UV $1.2 \pm 0.3$ ) and references (PP $2.7 \pm 0.7$, UV $1.9 \pm 0.9)(p<0.05)$. Conclusions: Abnormal mitochondrial distribution, a low capillary supply and signs of impaired oxidative activity suggest that muscle dysfunction of the palate muscles in long-term snorers may contribute to the upper airway obstruction during sleep. The cause of these abnormalities remains unclear, but local muscle and nerve trauma due to vibration and stretch is a possible etiology.

Copyright $\odot 2012$ S. Karger AG, Basel

\section{Introduction}

Snoring and obstructive sleep apnea syndrome (OSAS) is a part of the spectrum of sleep-disordered breathing (SDB) which includes all breathing abnormalities that occur during sleep. OSAS, characterized by repetitive episodes of upper airway obstruction, is common in the general population [1] and is associated with sleep fragmentation, hypersomnolence, increased cardiovascular risk and mortality [2]. Although obstruction may occur at several sites in the upper airways, the retropalatal region of the oropharynx is the most common site of collapse [3-5]. The pathophysiological mechanism of this col-

\section{KARGER}

Fax +41613061234

E-Mail karger@karger.ch

www.karger.com
(C) 2012 S. Karger AG, Basel

0025-7931/12/0835-0407\$38.00/0

Accessible online at:

www.karger.com/res
Dr. Per S. Stål

Department of Integrative Medical Biology

Section for Anatomy, Umeå University

SE-901 87 Umeå (Sweden)

Tel. +469078676 04, E-Mail per.stal@anatomy.umu.se 
lapse, and the obstruction that follows, is not fully understood but is considered to be multifactorial and includes both anatomical and physiological factors. Previous observations of an increase in connective tissue, abnormal muscle fiber size, changes in muscle fiber phenotypes [69], signs of nerve injury $[7,10]$ and inflammation [11] in upper airway muscles in patients indicate that an impaired upper airway muscle function might contribute to OSAS. Moreover, we have recently shown that the soft palate muscles in long-term snorers with OSAS have a reduced capillary supply [12]. This may cause disturbances in the microcirculation and local metabolism in the soft palate muscles and aggravate preexisting muscle dysfunction. In this study, we examined the intramyofibrillar organization and oxidative enzyme activity of mitochondria in relation to capillary supply and myosin heavy-chain (MyHC) isoform composition in palate muscles from patients with long-term snoring and upper airway obstruction.

\section{Patients and Methods}

\section{Patients}

Muscle biopsies from 8 patients, 7 males and 1 female (mean age $56 \pm 8$ years) were obtained at uvulopalatopharyngoplasty with or without tonsillectomy. The mean patient weight was $76 \pm$ $12 \mathrm{~kg}$, mean height $174 \pm 4 \mathrm{~cm}$ and mean body mass index $25 \pm$ 5. One patient was obese according to BMI criteria (BMI >30). Two were treated for arterial hypertension, but were otherwise healthy. All patients reported a long history of snoring with a mean duration of $17 \pm 13$ years. Overnight sleep registration confirmed snoring and apneas in all subjects. This registration included abdominal and thoracic movements, recordings of snoring sounds, registration of nasal/oral airflow with a thermistor, body movements and position by means of a static charge-sensitive bed and arterial oxygen saturation (BIOX 3740, ear probe; OMEDA Inc., Louisville, Colo., USA). In relation to estimated sleeping time, the mean percent of snoring was $33 \pm 18 \%$ and time in desaturation below $96 \%$ was $30 \pm 32 \%$, and below $90 \%$ it was $10 \pm$ $15 \%$. Demographic, clinical and morphological data of the subjects has already been published [12].

\section{Reference Muscles}

For comparison, control muscle samples from corresponding sites were obtained at an autopsy of 8 previously physically healthy subjects -6 males and 2 females with a mean age $51 \pm 10$ years - who had suffered a sudden accidental death. The specimens were obtained 1-2 days post mortem, a delay that does not affect nicotineamide adenine dinucleotide tetrazolium reductase (NADH-TR) or ATPase enzyme activity in muscle fibers [13]. BMI were available in 7 subjects, 6 were within normal limits (2025 ) and one was obese (31). The subject without BMI data had a normal body constitution according to the forensic rapport. All had normal craniofacial morphology and macroscopically nor- mal soft palate tissues. None of the subjects had been under medical treatment and there was no documented history of any significant disease, including SDB, alcohol or drug abuse. The autopsies did not reveal any previously unknown disease. The National Board of Health and Welfare, Stockholm, Sweden approved the use of the specimens which were collected in agreement with contemporary Swedish laws and regulations. All of our patients were informed about the purpose of the study and muscle samples were obtained from tissues removed at uvulopalatopharyngoplasty and after consent from the patients. All tissue samples were anonymous and without labeling or coding that would enable the identification of any living or dead person.

\section{Muscle Samples and Tissue Preparation}

After surgery, samples were obtained from resected parts of the palatopharyngeus (PP) and uvula (UV) muscles. The specimens were oriented for serial transverse sectioning in an OCT compound (Tissue $\mathrm{Tek}^{\circledR}$, Miles Laboratories, Naperville, Ill., USA), rapidly frozen in liquid propane chilled with liquid nitrogen and stored at $-80^{\circ} \mathrm{C}$ until further processing. No samples were stored in the freezer for more than 18 months before the staining process started.

\section{Enzyme Histochemistry}

Serial $10-\mu \mathrm{m}$-thick muscle cross sections were cut in a cryostat microtome at $-20^{\circ} \mathrm{C}$ and mounted on glass slides. The sections were stained for the mitochondrial enzymes, NADH-TR (EC1.6.99.3) and succinate dehydrogenase (SDH) (EC1.3.99.1) in order to demonstrate the oxidative activity and the intramyofibrillar distribution of mitochondria. The intramyofibrillarstained mitochondria in normal muscles are usually regularly ordered in a network with a uniform appearance throughout the individual fibers [14]. By using double histochemical staining for cytochrome c oxidase (COX) and SDH, deficits in the mitochondria respiratory chain were assessed. COX-positive fibers were stained brown and fibers without COX activity were blue. A modified Gomori trichrome (GT) staining was used to assess the general tissue morphology, pathology and accumulation of mitochondria. For the demonstration of myofibrillar ATPase activity (EC 3.6.1.3), the sections were stained for ATPase at $\mathrm{pH} 10.3,4.6$ and 4.3 .

\section{Immunohistochemistry}

The expression of MyHC isoforms was assessed on 5- $\mu \mathrm{m}$ thick muscle cross sections, serial to those used for enzyme histochemistry, by using previously well-characterized monoclonal antibodies (mAbs). Visualization of cell borders of muscle fibers and capillaries was performed with $\mathrm{mAbs}$ directed against laminin, a major noncollageneous component of the basement membrane. Monoclonal Ab 4C7 against the laminin $\alpha 5$-chain labels capillaries strongly and the basal lamina of muscle fibers weakly, whereas $\mathrm{mAb} 5 \mathrm{H} 2$ against the $\alpha 2$-chain labels only the basement membrane of muscle fibers. Both laminin mAbs have previously been used in the morphometric analysis of muscle capillaries and fiber areas $[12,15,16]$. For antibody specificity and origins, see table $1[15,17-24]$.

Immunostaining with $\mathrm{mAbs}$ was performed using standard techniques. The sections were first incubated in 5\% normal rabbit serum (Dako, Copenhagen, Denmark) for $15 \mathrm{~min}$ to inhibit subsequent unspecific staining, and then incubated with the primary 
Table 1. Data on antibodies used for immunohistochemistry

\begin{tabular}{|c|c|c|c|c|}
\hline Antibody & Specificity & Gene* & Source & Reference \\
\hline \multicolumn{5}{|l|}{$M y H C m A b s$} \\
\hline $\mathrm{A} 4.74$ & MyHCIIa & MYH2 & Hybridoma Bank, USA & Liu et al. [19], Hughes et al. [17] \\
\hline $\mathrm{N} 2.261$ & $\begin{array}{l}\text { MyHCI } \\
\text { MyHCIIa } \\
\text { MyHCeom } \\
\text { MyHC } \alpha \text {-cardiac }\end{array}$ & $\begin{array}{l}\text { MYH7 } \\
\text { MYH2 } \\
\text { MYH13 } \\
\text { MYH6 }\end{array}$ & Hybridoma Bank, USA & Liu et al. [19], Hughes et al. [17] \\
\hline F1.652 & MyHCembryonic & MYH3 & Hybridoma Bank, USA & Silberstein et al. [20] \\
\hline NCL-MHCn & MyHCfetal & MyH8 & Novocastra lab, Newcastle, UK & Weiss et al. [21], Ecob-Prince et al. [22] \\
\hline \multicolumn{5}{|l|}{ Laminin $m A b s$} \\
\hline $4 \mathrm{C} 7$ & laminin $\alpha 5$ chain & LAMA5 & Chemicon, Temecula, Calif., USA & Tiger et al. [23], Stål and Lindman [15] \\
\hline
\end{tabular}

mAbs diluted to appropriate concentrations with $0.01 \mathrm{M}$ phosphate-buffered saline (PBS) containing $0.1 \%$ bovine serum albumin for $1 \mathrm{~h}$ in a humid chamber at $37^{\circ} \mathrm{C}$. After washing in $0.01 \mathrm{M}$ PBS, the sections were incubated with normal rabbit serum for 15 min, followed by incubation with rabbit anti-mouse IgG (Dako) for $30 \mathrm{~min}$ at room temperature. The sections were washed in PBS and incubated with mouse peroxidase antiperoxidase (Dako) for $30 \mathrm{~min}$ and washed in PBS for $15 \mathrm{~min}$. The peroxidase binding was revealed by applying a solution containing diaminobenzidine (D5905 Sigma, St. Louis, Mo., USA), 0.05 M Tris buffer and hydrogen peroxidase $\left(\mathrm{H}_{2} \mathrm{O}_{2}\right)$ for 5-10 min. The sections were rinsed under running water, dehydrated in graded concentrations of ethanol followed by xylene treatment and finally mounted with DPX (BDH, Poole, UK). For the control of unspecific staining, sections were treated as described above, except that the incubation with the primary antibody was omitted. No staining was observed in these control sections.

\section{Fiber Phenotype Classification}

Based on the staining pattern for the different MyHC mAbs, the fibers were classified and grouped into fibers containing only slow MyHCI, only fast MyHCII (MyHCIIa and MyHCIIx) or as hybrid fibers coexpressing MyHCI and MyHCII. For control and comparison, the muscle fibers were enzyme-histochemically typed according to their staining intensities for myofibrillar ATPase (mATPase) after alkaline and acid preincubations [14]. Figure 1 summarizes the staining pattern for the classification of muscle fibers in different fiber phenotypes.

\section{Morphometric Analysis}

The muscle cross sections of each muscle and subject were positioned in a coordinate system and the muscle area was divided into four approximately equal-sized sections. By using the co-

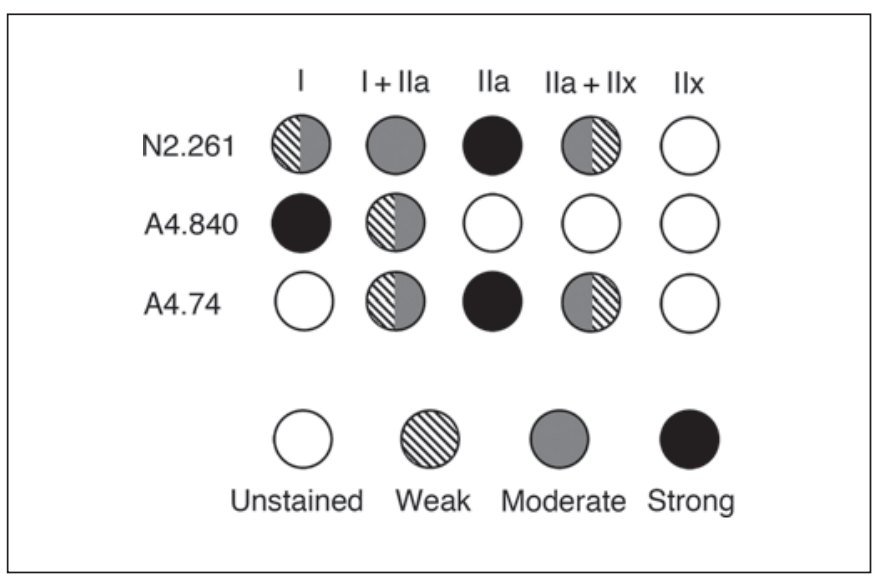

Fig. 1. Scheme of muscle fiber classification. The MyHC fiber phenotype classification is based on the mAbs staining pattern against different major contractile MyHC proteins. Monoclonal ab N2.261 is directed against MyHCIIa (strong staining) and MyHCI (weak staining), mAb A4.840 against MyHCI and mAb A4.74 against MyHCIIa. The various degrees of staining intensity are indicated. For the specificity of the different $\mathrm{mAbs}$, see table 1 .

ordinate system, one area from each of the four sections was randomly scanned $(\times 680)$ with a closed-circuit digital camera (DAGE-MTI, Michigan City, Ind., USA) connected to a light microscope (Zeiss Axiophot, Carl Zeiss, Oberkochen, Germany). The morphometric measurement was performed with an image analysis system (IBAS, Kontron Elektronik GmbH, Eching, Ger- 
Table 2. Proportion and fiber area of normal and different abnormal fibers in the PP and UV muscles of SDB patients and references

\begin{tabular}{llll}
\hline & $\begin{array}{l}\text { Subjects in } \\
\text { whom } \\
\text { present, } \mathrm{n}\end{array}$ & $\begin{array}{l}\text { Proportion } \\
\%\end{array}$ & $\begin{array}{l}\text { Fiber area } \\
\mu \mathrm{m}^{2}\end{array}$ \\
\hline PP muscle & & & \\
$\quad$ NAF & 8 & $61 \pm 22$ & $1,940 \pm 571$ \\
LT & 8 & $33 \pm 17$ & $1,535 \pm 379$ \\
SSM & 5 & $6 \pm 6$ & $645 \pm 363$ \\
ME & 2 & $0.3 \pm 0.6$ & N.m \\
COX-negative & 7 & $3.2 \pm 1.7$ & N.m \\
RR & 2 & $<0.1$ & N.m \\
Reference NAF & 8 & 100 & $1,643 \pm 569$ \\
\hline UV muscle & & & \\
NAF & 8 & $96 \pm 3$ & $1,647 \pm 281$ \\
LT & 8 & $3 \pm 3$ & $1,238 \pm 123$ \\
SSM & 1 & 1 & 413 \\
ME & 0 & $1.4 \pm 0.7$ & N.m \\
COX-negative & 4 & 100 & $1,200 \pm 271$ \\
RR & 0 & &
\end{tabular}

Values are expressed as mean values \pm standard deviations. N.m $=$ Not measured.

many). The staining activity for NADH-TR, SDH, COX-SDH and different MyHC mAbs in each fiber was analyzed and the circumference of each fiber and each capillary was traced along the periphery of the basement membrane in sections stained for mAbs against laminin (5H2 and 4C7). In the estimation of mitochondrial staining activity, fiber area, MyHC composition and fiber capillary supply, 1,939 individual muscle fibers were included. All measurements were performed by a single investigator blinded to the origin of the samples.

\section{Capillary Variables}

For the analysis of the number of capillaries around each fiber (CAF), all capillaries within a distance of $5 \mu \mathrm{m}$ from each muscle fiber were counted. Capillaries around fiber in relation to fiber cross-sectional area (CAFA) were calculated for each individual fiber according to the formula CAF/fiber cross-sectional area $\times$ $10^{3}$. The factor $10^{3}$ was used to obtain a number without many decimals and has no other mathematical importance.

\section{Statistics}

The Statview 4.5 (SAS Institute Inc., Cary, N.C., USA) statistical software was used. For comparing medians between groups, the Mann-Whitney U test was applied. The choice of a nonparametric test was based on the nonnormality in data distribution. In multiple comparisons, the Bonferroni correction was applied. The null hypothesis was rejected on p values $<0.05$.

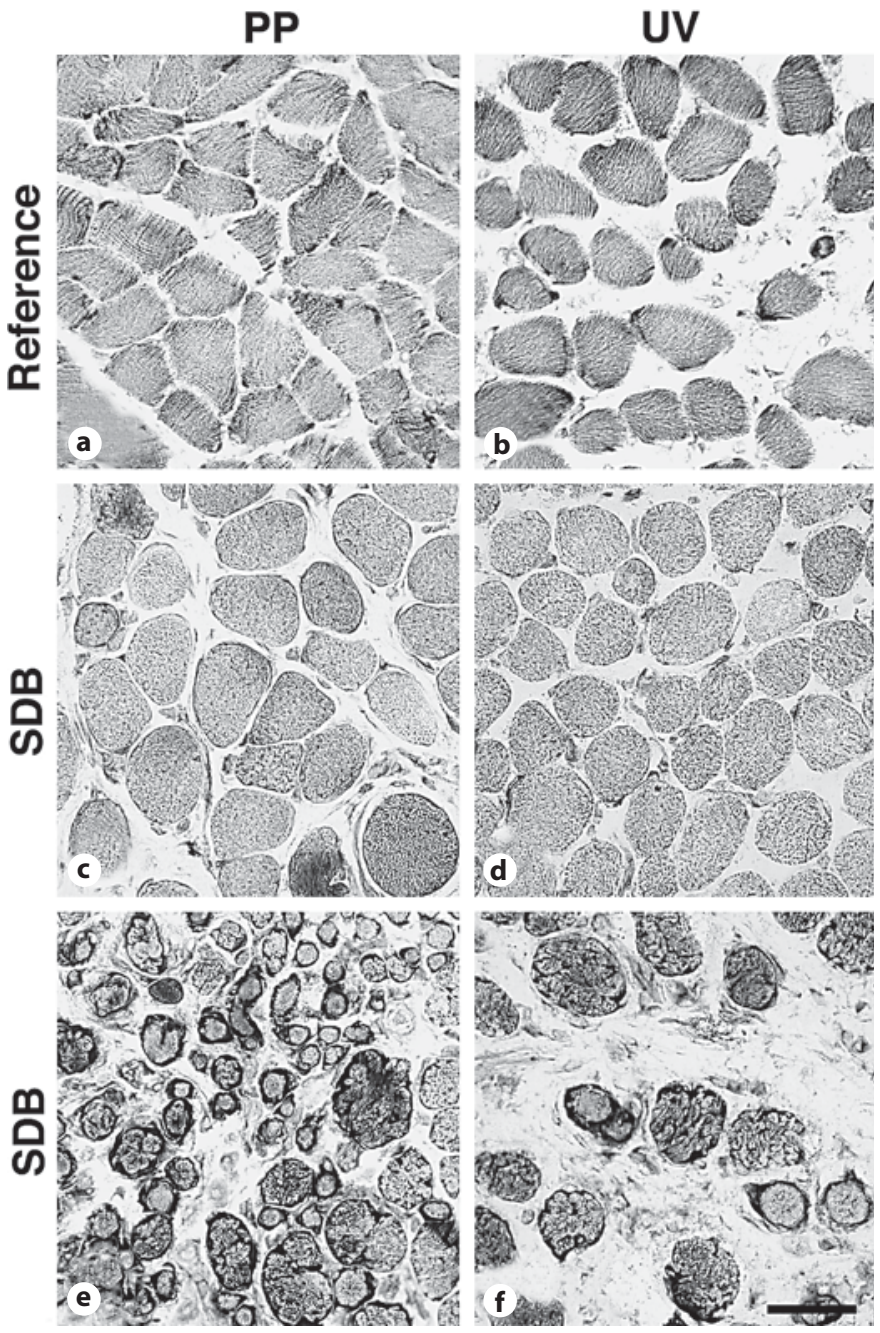

Fig. 2. Serial cross sections stained for NADH-TR from a PP (a, c, e) and $\operatorname{UV}(\mathbf{b}, \mathbf{d}, \mathbf{f})$ muscle from 1 reference subject $(\mathbf{a}, \mathbf{b})$ and 1 SDB patient (c-f). A normal intramyofibrillar mitochondria network is present in the reference muscles. Both $\operatorname{NAF}(\mathbf{c}, \mathbf{d})$ and fibers with abnormal mitochondria distribution $(\mathbf{e}, \mathbf{f})$ are shown in the muscles obtained from the SDB patient. Note the presence of fibers with a lobulated or rounded appearance, the large variability in fiber size and the extensive fibrosis in both the PP and UV muscles $(\mathbf{e}, \mathbf{f})$. f Scale bar $=50 \mu \mathrm{m}$.

\section{Results}

Mitochondria NADH-TR and SDH Enzyme Activity

Abnormal intramyofibrillar mitochondria staining patterns were found in all palate muscles from patients, but not in controls (table 2, fig. 2). Alterations in the myofibrillar staining activity and organization of mitochondria were observed in $39 \pm 15 \%$ (range $21-63 \%$ ) of fibers in the PP muscles and $4 \pm 3 \%$ (range 1-9\%) in the UV 
Fig. 3. Serial cross sections of a PP muscle stained for NADH-TR from a reference subject (a) and an SDB patient (b-d). a Normal mitochondria architecture in muscle fibers in a reference subject. b-d Examples of LT and SSM fibers in a PP muscle. Note the lobulated form, irregular trabecular and coarse mitochondria distribution and the large variability in size of the LT fibers. In the SSM fibers, there is subsarcolemmal accumulation of mitochondria and low mitochondria activity in the center (arrows). d Scale bar $=25 \mu \mathrm{m}$.
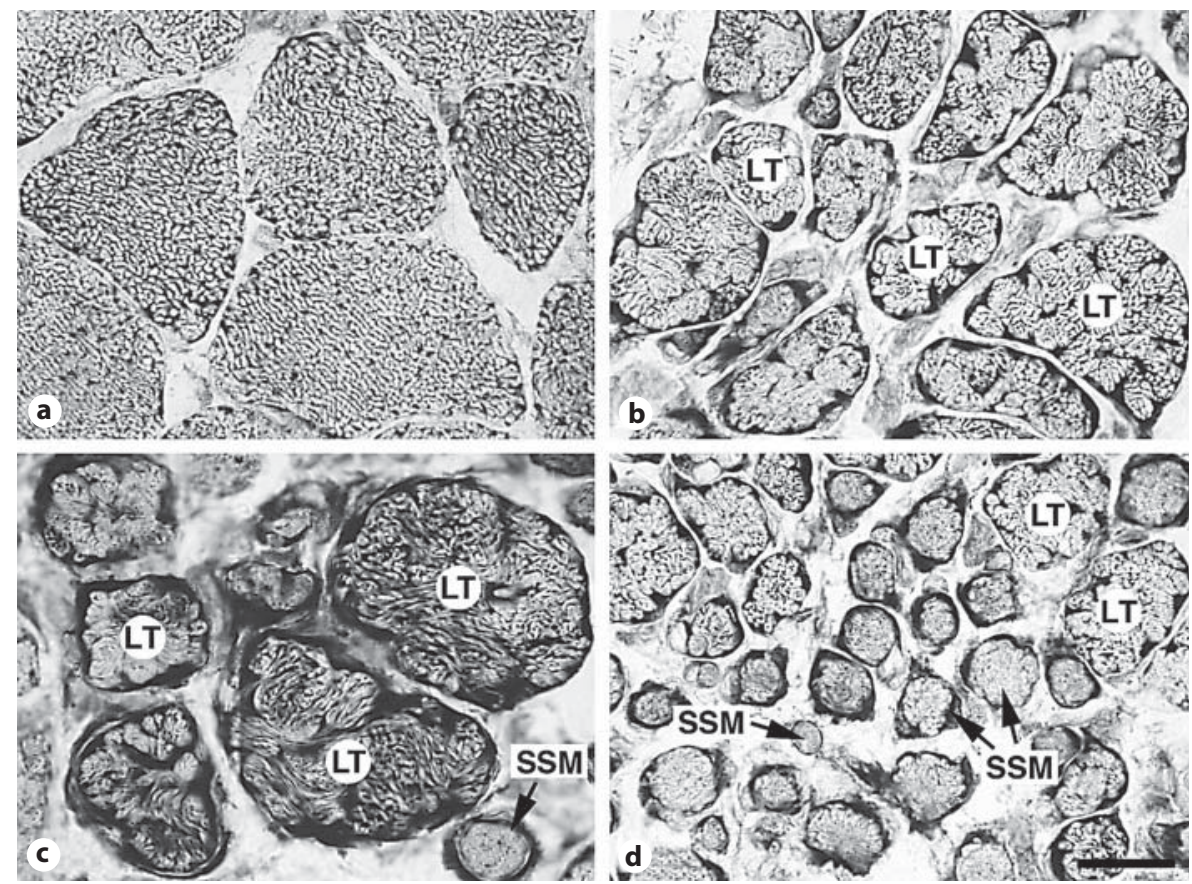

muscles. The abnormal fibers were usually distributed in clusters and in some cases involved complete fascicles. The most prevalent abnormality was an aberrant intramyofibrillar NADH-TR and SDH staining reaction with trabecular septations. The normal lattice pattern was replaced by a coarse granular structure with heterogeneous staining intensity, often interspersed with a conspicuous ring-like subsarcolemmal excess of reaction products. The vast majority of such fibers had a lobulated outer contour, but small fibers with a more rounded form and with low mitochondrial activity in the center were also common (fig. 2, 3). We will refer to fibers with a lobulated form and an irregular trabecular and coarse granular appearance as lobulated/trabecular (LT) fibers. The smaller and more rounded fibers with a dense subsarcolemmal accumulation of NADH-TR activity and absence or low activity towards the center will be termed small subsarcolemmal mitochondria (SSM) fibers (fig. 3). LT fibers were observed in all muscle samples from patients, and the proportion was higher in PP than in UV muscle (33 $\pm 17 \%$ vs. $3 \pm 3 \%, p<0.01)$. Fibers classified as SSM fibers were found in $5 \mathrm{PP}(6 \pm 6 \%)$ and $1 \mathrm{UV}$ muscle (1\%). Two of the PP samples contained occasional fibers $(0.3 \pm$ $0.6 \%$ ) with a staining pattern that corresponded to 'motheaten' (ME) fibers, i.e. fibers with normal contour but with irregular and multiple areas with a scarcity or lack of NADH-TR and SDH activity.

Abnormal Muscle Mitochondria

Distribution in Snorers with OSAS

\section{COX, GT and ATPase Enzyme Activity}

Fibers stained for SDH but unstained for COX were observed in a low proportion in $7 \mathrm{PP}$ and $4 \mathrm{UV}$ muscles $(3.2 \pm 1.7 \%$ vs. $1.4 \pm 0.7 \%)$. COX deficiency was observed in both normal fibers and fibers with abnormal mitochondrial distribution (table 2, fig. 4). In PP muscle sections stained for GT, occasional ragged-red (RR) fibers were observed $(<0.1 \%)$, i.e. fibers with a dense subsarcolemmal and intramyofibrillar accumulation of reddish material corresponding to the aggregations of formazan granula in NADH-TR [14]. The reddish-stained areas lacked activity in the corresponding areas in the sections stained for myofibrillar ATPase (table 2, fig. 5).

\section{Muscle Fiber Cross-Sectional Area}

In the PP muscle, the mean cross-sectional area of fibers with abnormal mitochondria organization was smaller than that of fibers with a normal appearance $\left(1,131 \pm 418 \mu \mathrm{m}^{2}\right.$ vs. $\left.1,920 \pm 524 \mu \mathrm{m}^{2}, \mathrm{p}<0.05\right)$. The rank in fiber size was in the PP muscle: SSM $<$ LT $<$ normal-appearance fibers (NAF) and in the UV muscle: SSM $<\mathrm{LT}=\mathrm{NAF}$. The SSM fibers were significantly smaller $(\mathrm{p}<0.05)$ than both LT fibers and normal cells in both $\mathrm{PP}\left(645 \pm 363 \mu \mathrm{m}^{2}\right.$ vs. $1,535 \pm 379 \mu \mathrm{m}^{2}$ and $1,940 \pm$ $571 \mu \mathrm{m}^{2}$, respectively) and UV muscle $\left(413 \pm 0 \mu \mathrm{m}^{2}\right.$ vs. $1,238 \pm 123 \mu \mathrm{m}^{2}$ and $1,647 \pm 281 \mu \mathrm{m}^{2}$, respectively) (fig. 6). 

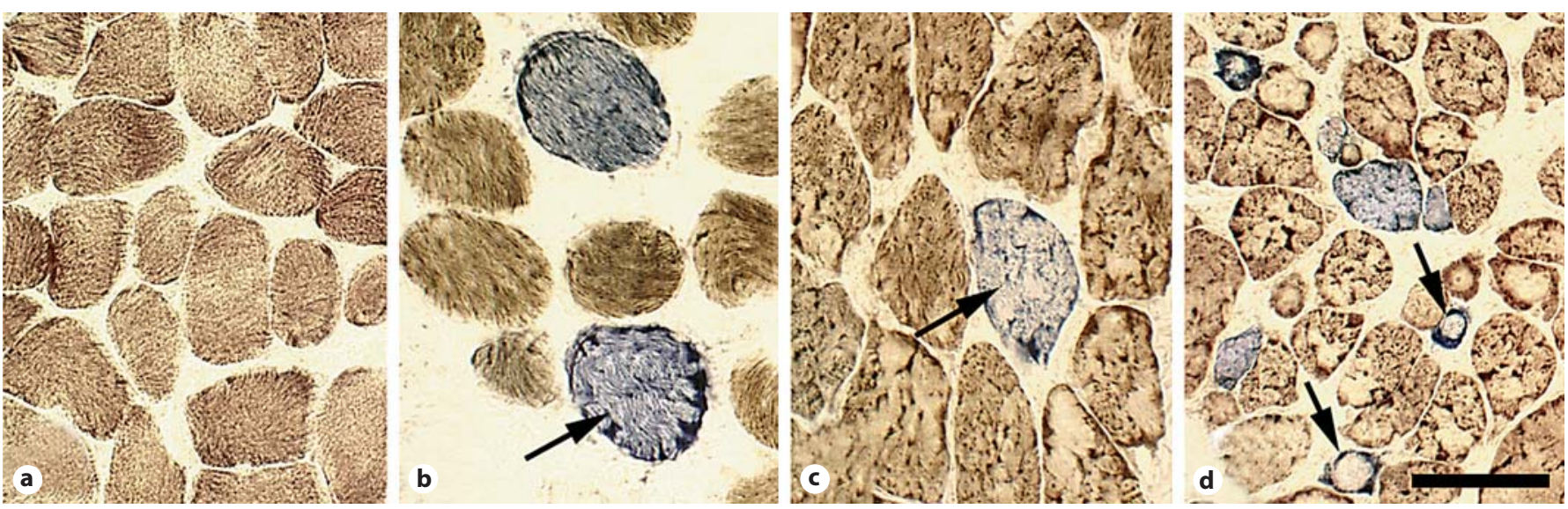

Fig. 4. Cross sections of a PP muscle double-stained for COX-SDH from a reference subject (a) and an SDB patient (b-d). COX-positive fibers are stained brown and COX-negative fibers are blue. Note the presence of COXnegative fibers (arrows) in both NAF (b), LT fibers (c) and SSM fibers (d) in the SDB subject. d Scale bar $=50 \mu \mathrm{m}$.
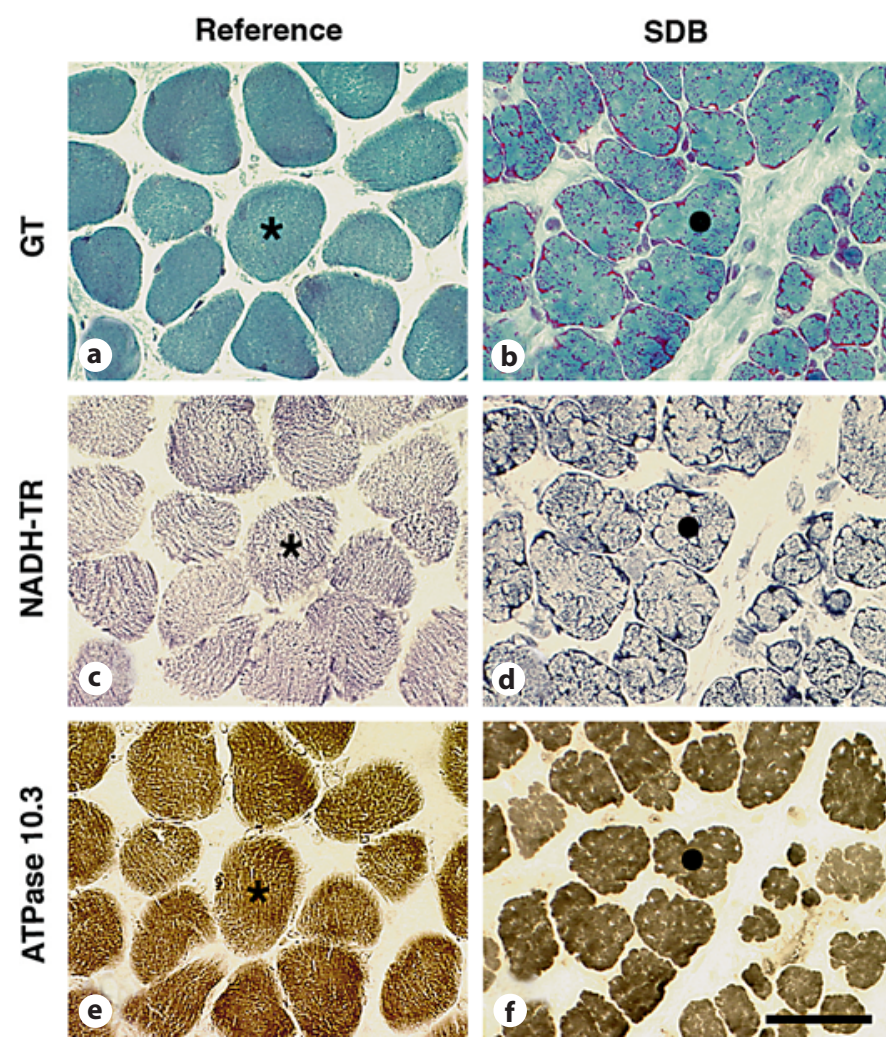

Fig. 5. Serial cross sections of the $\mathrm{PP}$ muscle from a reference subject $(\mathbf{a}, \mathbf{c}, \mathbf{e})$ and an SDB patient $(\mathbf{b}, \mathbf{d}, \mathbf{f})$ stained for GT $(\mathbf{a}, \mathbf{b})$, NADH-TR (c, d) and ATPase at pH $10.3(\mathbf{e}, \mathbf{f})$. In the SDB sample, note the strong SSM and LT fiber reaction in staining for GT (red areas) (b) and NADH-TR (dark blue areas) (d), and the lack of staining for ATPase in corresponding areas (f). The black point and the star denote the same fiber in the different sections. $f$ Scale bar $=50 \mu \mathrm{m}$.
Table 3. Proportion (\%) of fiber phenotypes (content of different $\mathrm{MyHCs}$ ) in fibers with abnormal mitochondria distribution in the palatopharyngeus and uvula muscles of $8 \mathrm{SDB}$ patients

\begin{tabular}{lccc}
\hline & MyHCI & MyHCI+II & MyHCII \\
\hline $\begin{array}{l}\text { Muscle } \\
\quad \text { Palatopharyngeus }\end{array}$ & $30 \pm 17$ & $16 \pm 14$ & $54 \pm 21$ \\
$\quad$ Uvula & $12 \pm 13$ & $6 \pm 1$ & $82 \pm 14$ \\
\hline
\end{tabular}

Values are expressed as mean \pm standard deviations.

Abnormal Mitochondria Organization in Relation to MyHC Composition and Capillary Supply

Fibers with an abnormal distribution of mitochondria were not related to a specific fiber phenotype or expression of developmental MyHC isoforms (table 3). The number of capillaries surrounding fibers with an abnormal distribution of mitochondria was lower than those surrounding the NAF, in both PP (CAF $0.9 \pm 0.3$ vs. 1.4 $\pm 0.6, \mathrm{p}<0.01)$ and $\mathrm{UV}(\mathrm{CAF} 0.4 \pm 0.1$ vs. $1.2 \pm 0.3$, $\mathrm{p}<0.05)$. However, when compensating for the differences in fiber area (CAFA), no statistical difference in normal cells was observed (fig. 7).

\section{Other Histopatholgical Findings}

The most common morphological and histopathological palate muscle findings were fibrosis, marked variability in fiber size and an increased proportion of smallsized fibers expressing developmental $\mathrm{MyHC}$ isoforms. 


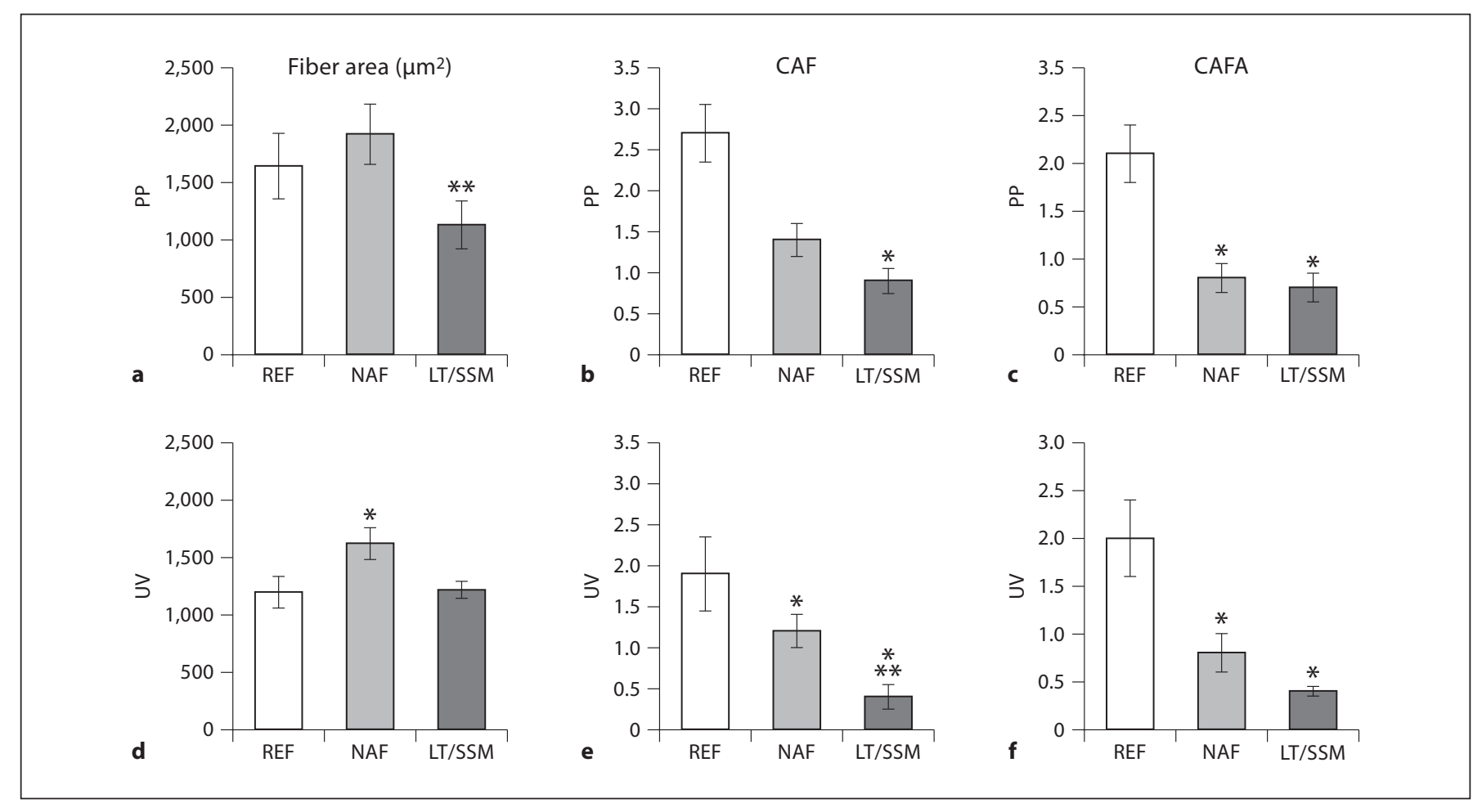

Fig. 6. Graphs visualizing the mean fiber area in $\mu \mathrm{m}^{2}(\mathbf{a}, \mathbf{d}), \operatorname{CAF}(\mathbf{b}, \mathbf{e})$ and CAFA (c, f) in the PP (a-c) and UV (d-f) muscles from reference subjects (REF) and SDB patients. Standard deviations (error bars) are indicated. ${ }^{*} \mathrm{p}<0.05$ = significantly different from REF; ${ }^{* *} \mathrm{p}<0.05=$ significantly different from NAF.

These features were frequently observed in PP, and to a lesser extent in UV. Whereas both fiber atrophy and hypertrophy were common in PP, fiber hypertrophy dominated in the UV. Clusters of small fibers, i.e. fiber group atrophy, were observed in the PP but generally not in UV (fig. 1, 2).

\section{Comparison to Control Muscles}

In the controls except for occasional fibers, there was a normal mitochondrial staining pattern for NADH-TR and SDH within the muscle fibers in both the PP and UV muscles (fig. 2-5). Fiber size and fiber form were more homogeneous than in the patients and the muscle:connective tissue ratio higher. Only occasional fibers were stained for developmental MyHCs and only a few COX-negative fibers were observed in one of the controls. No fibers corresponding to the criteria for moth-eaten or ragged-red fibers were detected. The area of fibers in the PP muscles did not differ significantly from normal fibers in patients, but were larger than fibers with irregular mitochondria organization $\left(1,643 \pm 569 \mu \mathrm{m}^{2}\right.$ vs. 1,131 $\left.\pm 298 \mu \mathrm{m}^{2}, \mathrm{p}<0.05\right)$. In contrast, fibers in the UV mus- cles did not differ in size from fibers with disturbed mitochondria organization and the area was smaller than that of NAF in the patients $\left(1,200 \pm 271 \mu \mathrm{m}^{2}\right.$ vs. $1,623 \pm$ $298 \mu \mathrm{m}^{2}, \mathrm{p}<0.05$ ) (table 2).

The number of capillaries supplying an individual fiber was higher in the control PP (CAF $2.7 \pm 0.7$ ) and UV (CAF $1.9 \pm 0.9$ ) compared to both normal and abnormal fibers in the patient samples $(\mathrm{p}<0.05)$. After correction for differences in fiber area, the capillary supply was still higher in controls for both PP (CAFA $2.1 \pm 0.6)$ and UV (CAFA $2.0 \pm 0.8$ ) muscles (fig. 6, 7).

\section{Discussion}

In this study we show that soft palate muscles in patients with long-term snoring and upper airway obstruction contain a high proportion of fibers with an abnormal internal distribution and oxidative enzyme-staining activity of mitochondria, a finding in support of our previous reports on neuromuscular injury as a possible mechanism for upper airway muscle dysfunction in this dis- 

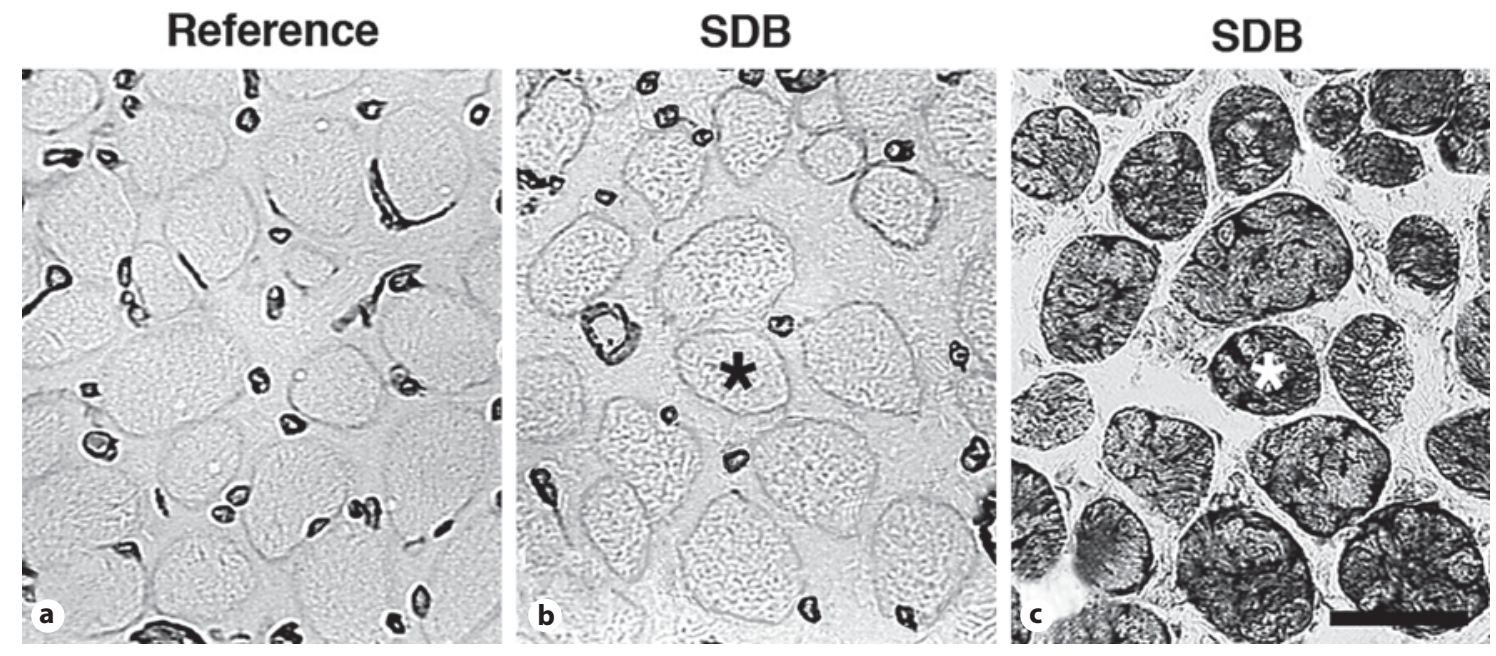

Fig. 7. Cross sections from PP muscle from a reference subject (a) and an SDB patient (b, c) stained for laminin $\alpha 5$-chain (a, b) and NADH-TR (c). b, c Serial sections in which the star denotes the same muscle fiber. The sections show stained capillaries in a reference subject (a) and around LT fibers in an SDB patient (b). Note the lower capillary density in the SDB patient than in the reference subject. c Scale bar $=50 \mu \mathrm{m}$.

ease $[9,12]$. Dysfunction of the upper airway muscles might cause an imbalance between forces acting to keep the airway open and negative inspiratory airway pressure that causes obstruction of the pharynx during sleep. Together with a decreased capillary supply and other histopathological muscle changes, our results suggest that muscle injury, including metabolic disturbances, may be a part of the pathophysiology in this disease.

The most common morphological abnormality was clusters of fibers with a lobulated contour and an internal irregular distribution of stained mitochondria, often in a trabecular pattern. The higher proportion of these fibers, i.e. LT fibers, was more evident in the PP muscle (33\%) than in the UV muscle (4\%). Fibers displaying a lobulated and trabecular appearance have been reported in various neuromuscular diseases and this is considered a nonspecific reaction $[25,26]$ or a typical finding in certain forms of muscular dystrophies [26-28]. Despite the frequent presence of lobulated/trabecular fibers in various muscle disorders, the significance of these fibers is still unknown. The abnormal distribution of mitochondria might, however, cause an uneven intracellular distribution of energy production that per se can have negative effects on myocyte function.

Small and rounded fibers with subsarcolemmal dense aggregates of mitochondria and an aberrant organization or focal loss of stained mitochondria in the more central parts, i.e. SSM fibers, were also relatively common in the
PP muscle (6\%). The pattern of mitochondrial distribution and the small area of SSM fibers, in addition to the more prevalent lobulated trabecular fibers, are in line with findings in limb-girdle dystrophy [26] and indicate fiber degeneration and/or fiber regeneration. However, the majority of these fibers did not contain embryonic or fetal MyHC, two markers for the early stages of muscle fiber development and regeneration [29, 30], suggesting that the SSM fibers observed were more likely involved in a degenerative rather than in a regenerative process. The distribution in clusters or presence in whole fascicles, suggests a process of continuous loss of motor innervation and insufficient reinnervations. Thus, the differences between SSM and LT fibers in mitochondrial organization and size might be related to the different stages on a spectrum of myocyte reaction to injury.

The finding that LT and SSM fibers were preferentially found in fibers expressing fast MyHCII in the SDB subjects might to some extent be explained by the distinct predominance of fast phenotype fibers (MyHCII) in normal PP (69\%) and UV (86\%) muscles [15]. An alternative is that the mitochondria compartment in fibers expressing $\mathrm{MyHCII}$ is more sensitive for other factors, such as disturbances in microcirculation or denervation. A support for the latter is that fibers expressing MyHCI, normally more highly oxidative and better supplied by capillaries than fibers expressing MyHCII, seem to be less prone to atrophy in the early stages of muscle denervation [31]. 
Fibers with another type of disturbed myofibrillar network, so-called moth-eaten fibers, have been reported to be frequently present in palate muscles samples from SDB patients $[6,7]$. In limb muscles, these fibers are particularly seen in various myopathies as a nonspecific finding. However, in our study, moth-eaten fibers were rare. The contrasting results might be related to the different criteria for defining fibers with a disturbed mitochondria intramyofibrillar network or to different sample areas.

Fibers lacking staining for COX, the complex IV of the mitochondria respiratory chain, were present in all patients except one (PP 3.2\%, UV 1.7\%). In contrast, only one control sample contained occasional COX-negative fibers. The increased presence of COX-deficient fibers in young and middle-aged adults is considered to be a hallmark of mitochondrial disease [32]. Abnormalities in the complex IV respiratory chain decrease the oxidative capacity of muscle fibers and impair the contractile force and fatigue resistance. However, a small number of COXdeficient fibers $(1-2 \%)$ is not uncommon in patients over 65 years of age, owing to age-related somatic DNA mutations or gene deletions possibly due to the accumulation of oxygen free radicals [33]. The significance of COX-deficient fibers in the palate muscles of SDB patients is unclear, but neuromuscular damage and/or inflammation might increase oxygen-free radicals over time, leading to mitochondrial DNA mutations in the upper airway muscles $[34,35]$.

A few fibers with a ragged-red appearance were observed among the LT fibers in 2 of the patients. This is a characteristic finding in various mitochondrial diseases and a hallmark of morphological changes due to disturbed oxidative phosphorylation [36]. Ragged-red fibers increase with age and are frequently observed in inflammatory myopathy $[35,37]$. Although the presence of COX-deficient fibers, moth-eaten and ragged-red fibers further suggests the presence of bioenergetically deficient fibers in the upper airway muscles of SDB patients, the proportion is probably too low to be of important functional significance. However, their presence still supports alterations in the mitochondrial compartment as part of the pathological process in SDB.

The muscle abnormalities observed in this study may hypothetically be a consequence of vibratory trauma and/ or traumatic stretching of soft palate tissues during snoring. Numerous studies in occupational medicine have shown links between vibration and tissue injury in limb muscles. Long-standing exposure to vibrations causes a loss of vibrotactile sensation, local nerve lesions, muscle fiber damage [38-40], loss of capillaries [41], vasospasm [42], a decreased blood flow [43, 44] and decreased muscle strength [45]. Muscle ischemia and vascular disease that affect capillaries have been reported to cause a gradual deterioration of the mitochondrial organization in muscle $[46,47]$. Thus, based on the consequence of vibratory trauma, long-standing snoring vibrations might create a substrate for hypoxia and an energy crisis that may lead to a vicious circle of injury and further functional impairment in the mitochondria compartment. Moreover, overuse and repeated muscle contractions during active muscle lengthening of the upper airway can induce myofibrillar disturbances, a loss of mitochondria and a decrease in force-generating capacity [48, 49]. Several other biological processes may be responsible for the abnormal spatial mitochondrial distribution. However, irrespective of the cause, it is reasonable to consider that the abnormalities we and others observed may affect the function of upper airways.

The discrepancy in abnormalities between the PP and UV muscles from SDB patients is of specific interest. Whereas PP muscles were characterized by severe abnormalities, the UV muscles had relatively few abnormalities and were characterized by a high proportion of fibers with a larger mean cross-sectional area than in controls, i.e. muscle fiber hypertrophy. This indicates an adaptation to meet the additional load on the muscle to maintain the airway patency during inspiration. In contrast, the frequent abnormalities including both fiber hypertrophy and fiber atrophy in the PP muscle suggest a more pronounced process of both degeneration and regeneration in this muscle. However, although snoring vibrations/stretch of palate tissue or traumatic muscle contractions had different morphological effects on the muscles, both muscle groups had a generally lower NADH-TR activity and a lower capillary supply compared to the reference muscles, which indicates a decreased endurance capacity. When gravity negatively influences the width of the upper airway and the tonic activity of the pharyngeal muscles decrease during sleep [50], a diminished endurance capacity might increase the risk for the collapse of pharyngeal walls during inspiration.

The cause of the differences in morphological alterations between the PP and UV muscles of SDB patients is unclear. This could be related to their anatomical localization in the pharynx, orientation and insertions of muscle fibers, or significance in dilating the upper airways. The UV muscle is located parallel to the airstream in the center of the soft palate, whereas the PP muscle is oriented transversely to the airstream laterally and super- 
ficially in the palatopharyngeal arches. During snoring, the vigorous vibration may be more frequent and traumatic in the loose posterior and lateral parts of the soft palate where the PP muscle is located. Moreover, since the PP muscle has attachments at both ends and the UV muscle at only one end, the strain on the muscle might be higher in the PP during contraction in an attempt to terminate apneas, which may increase the risk for injury.

A limitation of our study is that despite no history of snoring or OSAS being reported in the controls, this cannot be excluded. However, since all palate muscle samples from the controls showed normal muscle morphology and a normal intramyofibrillar distribution of mitochondria, which is in clear contrast to the samples from the SDB patients (where all the muscles were affected), we assume that the reference muscle samples are representative as controls without significant SDB.

Altogether, the findings of an abnormal mitochondrial distribution and a reduced capillary supply in the SDB palate muscles, together with our previous observations of marked structural abnormalities [12, 15], strongly in- dicate that a neuromuscular and metabolic component contributes to the instability and collapse of the upper airway muscles in SDB. Although there are signs of adaptive processes to strengthen the muscles, dysfunction of both contractile and energy-supplying compartments might result in reduced endurance and muscle weakness and thereby affect overall muscle function. If there is a vicious circle where snoring induces muscle and nerve injury which leads to worse oropharyngeal muscle function and deterioration of SDB, a therapy targeting SDB, e.g. continuous positive airway pressure, may have the potential to break this vicious circle and inhibit or delay further muscle injury.

\section{Acknowledgements}

This work was supported by grants from the Swedish HeartLung Foundation and the Heart Foundation of Northern Sweden. We thank Dr. Lars Ödqvist and Dr. Rolf Lindman for valuable support and Anna-Karin Olofsson, Mona Lindström and Ulrica Högbacka for their technical assistance.

\section{References}

1 Young T, Palta M, Dempsey J, Skatrud J, Weber S, Badr S: The occurrence of sleep-disordered breathing among middle-aged adults. N Engl J Med 1993;328:1230-1235.

-2 Dempsey JA, Veasey SC, Morgan BJ, O'Donnell CP: Pathophysiology of sleep apnea. Physiol Rev 2010;90:47-112.

3 Hudgel DW: Mechanisms of obstructive sleep apnea. Chest 1992;101:541-549.

4 Shepard JW Jr, Thawley SE: Localization of upper airway collapse during sleep in patients with obstructive sleep apnea. Am Rev Respir Dis 1990;141:1350-1355.

$\checkmark 5$ Morrison DL, Launois SH, Isono S, Feroah TR, Whitelaw WA, Remmers JE: Pharyngeal narrowing and closing pressures in patients with obstructive sleep apnea. Am Rev Respir Dis 1993; 148:606-611.

-6 Edström L, Larsson H, Larsson L: Neurogenic effects on the palatopharyngeal muscle in patients with obstructive sleep apnoea: a muscle biopsy study. J Neurol Neurosurg Psychiatry 1992;55:916-920.

-7 Friberg D, Ansved T, Borg K, CarlssonNordlander B, Larsson H, Svanborg E: Histological indications of progressive snorer's disease in an upper airway muscle. Am J Respir Crit Care Med 1998;157:586-593.

8 Friberg D: Heavy snorer's disease: a progressive local neuropathy. Acta Otolaryngol 1999; 119:925-933.
-9 Lindman R, Stål PS: Abnormal palatopharyngeal muscle morphology in sleep-disordered breathing. J Neurol Sci 2002;195:1123.

10 Woodson BT, Garancis JC, Toohill RJ: Histopathologic changes in snoring and obstructive sleep apnea syndrome. Laryngoscope 1991;101:1318-1322.

11 Boyd JH, Petrof BJ, Hamid Q, Fraser R, Kimoff RJ: Upper airway muscle inflammation and denervation changes in obstructive sleep apnea. Am J Respir Crit Care Med 2004;170: 541-546.

12 Stål PS, Lindman R, Johansson B: Capillary supply of the soft palate muscles is reduced in long-term habitual snorers. Respiration 2009;77:303-310.

13 Eriksson PO, Eriksson A, Ringqvist M, Thornell LE: The reliability of histochemical fibre typing of human necropsy muscles. Histochemistry 1980;65:193-205.

14 Dubowitz V, Sewry A: Muscle Biology. A Practical Approach, ed 3. London, Saunders Elsevier, 2007.

15 Stål PS, Lindman R: Characterisation of human soft palate muscles with respect to fibre types, myosins and capillary supply. J Anat 2000;197:275-290.
16 Pontén EM, Stål PS: Decreased capillarization and a shift to fast myosin heavy chain IIx in the biceps brachii muscle from young adults with spastic paresis. J Neurol Sci 2007; 253:25-33.

17 Hughes SM, Cho M, Karsch-Mizrachi I, Travis $\mathrm{M}$, Silberstein $\mathrm{L}$, Leinwand LA, Blau HM: Three slow myosin heavy chains sequentially expressed in developing mammalian skeletal muscle. Dev Biol 1993;158:183-199.

18 Cho M, Webster SG, Blau HM: Evidence for myoblast-extrinsic regulation of slow myosin heavy chain expression during muscle fiber formation in embryonic development. J Cell Biol 1993;121:795-810.

19 Liu JX, Eriksson PO, Thornell LE, PedrosaDomellof F: Myosin heavy chain composition of muscle spindles in human biceps brachii. J Histochem Cytochem 2002;50:171183.

20 Silberstein L, Webster SG, Travis M, Blau HM: Developmental progression of myosin gene expression in cultured muscle cells. Cell 1986;46:1075-1081.

21 Weiss A, Schiaffino S, Leinwand LA: Comparative sequence analysis of the complete human sarcomeric myosin heavy chain family: implications for functional diversity. J Mol Biol 1999;290:61-75. 
22 Ecob-Prince M, Hill M, Brown W: Immunocytochemical demonstration of myosin heavy chain expression in human muscle. J Neurol Sci 1989;91:71-78.

$\checkmark 23$ Tiger CF, Champliaud MF, PedrosaDomellöf F, Thornell LE, Ekblom P, Gullberg D: Presence of laminin alpha 5 chain and lack of laminin alphal chain during human muscle development and in muscular dystrophies. J Biol Chem 1997;272:28590-28595.

-24 Sewry CA, Philpot J, Mahony D, Wilson LA, Muntoni F, Dubowitz V: Expression of laminin subunits in congenital muscular dystrophy. Neuromuscul Disord 1995;5:307-316.

$>25$ Bethlem J, van Wijngaarden GK, de Jong J: The incidence of lobulated fibres in the facioscapulo-humeral type of muscular dystrophy and the limb-girdle syndrome. J Neurol Sci 1973;18:351-358.

-26 Weller B, Carpenter S, Lochmuller H, Karpati G: Myopathy with trabecular muscle fibers. Neuromuscul Disord 1999;9:208-214.

-27 Figarella-Branger D, El-Dassouki M, Saenz A, Cobo AM, Malzac P, Tong S, Cassotte E, Azulay JP, Pouget J, Pellissier JF: Myopathy with lobulated muscle fibers: evidence for heterogeneous etiology and clinical presentation. Neuromuscul Disord 2002;12:4-12.

28 Guerard MJ, Sewry CA, Dubowitz V: Lobulated fibers in neuromuscular diseases. J Neurol Sci 1985;69:345-356.

-29 Butler-Browne GS, Whalen RG: Myosin isozyme transitions occurring during the postnatal development of the rat soleus muscle. Dev Biol 1984;102:324-334.

30 Banker BQ, Engel AG: Myology, ed 2. New York, Mc Graw-Hill, 1994.
31 Borisov AB, Huang SK, Carlson BM: Remodeling of the vascular bed and progressive loss of capillaries in denervated skeletal muscle. Anat Rec 2000;258:292-304.

32 Taylor RW, Schaefer AM, Barron MJ, McFarland R, Turnbull DM: The diagnosis of mitochondrial muscle disease. Neuromuscul Disord 2004; 14:237-245.

33 Johannsen DL, Ravussin E: The role of mitochondria in health and disease. Curr Opin Pharmacol 2009;9:780-786.

34 Lavie L: Obstructive sleep apnoea syndrome - an oxidative stress disorder. Sleep Med Rev 2003;7:35-51.

35 Chariot P, Ruet E, Authier FJ, Labes D, Poron F, Gherardi R: Cytochrome c oxidase deficiencies in the muscle of patients with inflammatory myopathies. Acta Neuropathol 1996;91:530-536.

36 Nardin RA, Johns DR: Mitochondrial dysfunction and neuromuscular disease. Muscle Nerve 2001;24:170-191.

37 Rifai Z, Welle S, Kamp C, Thornton CA: Ragged red fibers in normal aging and inflammatory myopathy. Ann Neurol 1995;37: 24-29.

38 Takeuchi T, Futasuka M, Imanishi H, Yamada S: Pathological changes observed in the finger biopsy of patients with vibration-induced white finger. Scand J Work Environ Health 1986;12:280-283.

39 Koskimies K, Farkkila M, Pyykko I, Jantti V, Aatola S, Starck J, Inaba R: Carpal tunnel syndrome in vibration disease. Br J Ind Med 1990;47:411-416.

40 Virokannas H: Vibration perception thresholds in workers exposed to vibration. Int Arch Occup Environ Health 1992;64:377382 .
41 Murfee WL, Hammett LA, Evans C, Xie L, Squire M, Rubin C, Judex S, Skalak C: Highfrequency, low-magnitude vibrations suppress the number of blood vessels per muscle fiber in mouse soleus muscle. J Appl Physiol 2005;98:2376-2380.

42 Stoyneva Z, Lyapina M, Tzvetkov D, Vodenicharov E: Current pathophysiological views on vibration-induced Raynaud's phenomenon. Cardiovasc Res 2003;57:615624

43 Fukagawa Y, Otsu M, Nakano M, Maki T: Analysis of capillary fluid flow rate by vibration on a small vessel model. J UOEH 1986; 8:159-168.

44 Curry BD, Bain JL, Yan JG, Zhang LL, Yamaguchi M, Matloub HS, Riley DA: Vibration injury damages arterial endothelial cells. Muscle Nerve 2002;25:527-534.

45 Farkkila M: Grip force in vibration disease. Scand J Work Environ Health 1978;4:159166.

46 Heffner RR BS: The early effects of ischemia upon skeletal muscle mitochondria. J Neurol Sci 1978;38:295-315.

47 Carpenter S KG: Pathologhy of skeletal muscle. New York, Churchill Livingstone, 1984.

48 Clarkson PM, Hubal MJ: Exercise-induced muscle damage in humans. Am J Phys Med Rehabil 2002;81:S52-S69.

49 Fridén J, Sjöström M, Ekblom B: Myofibrillar damage following intense eccentric exercise in man. Int J Sports Med 1983;4:170-176.

50 Horner RL: Pathophysiology of obstructive sleep apnea. J Cardiopulm Rehabil Prev 2008;28:289-298. 